\title{
$X$-band rf structure with integrated alignment monitors
}

\author{
M. Dehler, J.-Y. Raguin, A. Citterio, and A. Falone \\ PSI, Villigen PSI, Switzerland \\ W. Wuensch, G. Riddone, A. Grudiev, and R. Zennaro \\ CERN, Geneva, Switzerland \\ (Received 30 January 2009; published 3 June 2009)
}

\begin{abstract}
We present the electrical design for an $X$-band traveling wave accelerator structure with integrated alignment monitors to measure the transverse wake, which will be used as part of the PSI-XFEL project and in the CLIC structure testing program. At PSI, it will compensate nonlinearities in the longitudinal phase space at the injector prototype of the PSI-XFEL. At CLIC it will be tested for breakdown limits and rates in the high gradient regime. The prolonged operation of such a structure in the PSI-XFEL injector, albeit not for the CLIC parameter regime, will constitute a good quality test of the manufacturing procedures employed. The operation in the PSI-XFEL injector will be at a relatively modest beam energy of $250 \mathrm{MeV}$, at which transverse wakes can easily destroy the beam emittance. For this reason, the layout chosen employs a large iris, $5 \pi / 6$ phase advance geometry, which minimizes transverse wakefield effects while still retaining a good efficiency. As a second important feature, the design includes two wakefield monitors coupling to the transverse higher order modes, which allow steering the beam to the structure axis, potentially facilitating a higher precision than mechanical alignment strategies. Of special interest is the time domain envelope of these monitor signals. Local offsets due to bends or tilts have individual signatures in the frequency spectrum, which in turn are correlated with different delays in the signal envelope. By taking advantage of this combined with the single bunch mode at the PSI-XFEL, the use of a relatively simple detector-type rf front end should be possible, which will not only show beam offsets but also higher order misalignments such as tilts in the structure. The resolution of these monitors is determined by the tolerance of the random cell-to-cell misalignment leading to a spurious signal in the monitors.
\end{abstract}

DOI: 10.1103/PhysRevSTAB.12.062001

PACS numbers: 29.20.Ej

\section{INTRODUCTION}

Within the context of the JLC-X (later named GLC) and NLC projects, a considerable effort had been going on in developing high power $X$-band rf systems for high energy $e^{+} e^{-}$colliders [1]. After the termination of these projects, the recent decision by CLIC to change their principal rf frequency from $30 \mathrm{GHz}$ to a European $X$-band frequency near $12 \mathrm{GHz}$ has given a renewed substantial push to $X$-band development. An rf structure fabrication and testing program involving major laboratories around the world is under way; research and development efforts have been directed toward adapting standard rf equipment, from high power klystrons to splitters and loads, to the new frequency.

Interestingly, $X$-band technology is slowly becoming of interest for other applications. The LCLS FEL [2] uses an $X$-band structure to compensate nonlinearities in the longitudinal phase space in order to improve bunch compression. The European FEL projects SPARC [3], FERMI@ELETTRA [4], and PSI-XFEL [5] plan to employ $X$-band $\mathrm{rf}$ for the same purpose. There is also a discussion going on to use $X$ band for compact medical accelerators [6].
Whereas the SPARC project is opting for rf structures at the NLC/GLC frequency of $11.424 \mathrm{GHz}$, PSI and FERMI have decided to follow the new European standard at $11.992 \mathrm{GHz}$. For the PSI-XFEL, this choice allows an optional future operation in a few bunch or multibunch operation mode (given that the current setup uses already European $S$ and $L$ band). In that context, a klystron is currently under development by SLAC, an adapted version of type XL4 [7] to be christened XL5. The X-band structure is being developed within a collaboration between PSI and CERN, optimizing synergy on both sides. The structure will serve several purposes, being submitted to breakdown tests at CERN, and being used in the PSI-XFEL, which constitutes, despite different operating parameters, a long duration performance test of some importance for CLIC.

At the PSI-XFEL, we have only limited power, on the order of $40 \mathrm{MW}$ at the structure after taking account of waveguide losses, and need to generate beam voltages up to $30 \mathrm{MeV}$, which requires a fairly efficient structure. But the beam has a relatively modest energy of $250 \mathrm{MeV}$, so that we are relatively sensitive to transverse wakes. Therefore the structure needs to be a good compromise between a high shunt impedance, generally associated with 
low apertures, and a low transverse kick, demanding the opposite.

As a generic type, we use a structure with a $5 \pi / 6$ phase advance and an active length of $750 \mathrm{~mm}$, similar to NLC type $\mathrm{H} 75$ [8]. The structure will not be used to test higher order mode (HOM) damping; hence, it will not have any HOM dampers. Nonetheless, two wakefield monitors near the beginning and the end of the structure are foreseen, which will help to align the structure to the beam and to minimize transverse kicks. Wakefield monitors are also planned for CLIC structures, and their implementation in the PSI-FEL structures will represent an important performance test.

The monitor uses a TE-type coupling to the hybrid electromagnetic (HEM) dipole modes in the cavity, offering a very good rejection of the fundamental as well as higher order longitudinal modes. The constant gradient design gives a variation of the synchronous dipole frequency through the structure, so that the signals contain more information than only the simple offset. This feature has already been exploited in other setups by doing a spectral analysis of the signal. Here we also make use of the fact that wave propagation inside the rf structure is relatively slow, only a few percent of the speed of light, so that similar details can also be seen in the spread out pulse response of the wake monitors. Relatively simple rf front ends can be used, giving only the envelope of the output signal.

\section{FUNDAMENTAL MODE PROPERTIES}

For the PSI-XFEL, a single structure with $750 \mathrm{~mm}$ active length is the most appropriate solution in terms of simplicity and cost. The NLC structure type H75 $(5 \pi / 6$ phase advance) has been chosen as the most suitable candidate for the testing program. The original H75 design, optimized for iris aperture, thickness and ellipticity of the iris varying along the structure, provides an accelerating gradient of $65 \mathrm{MV} / \mathrm{m}$ for $80 \mathrm{MW}$ input power and was successfully tested up to $100 \mathrm{MV} / \mathrm{m}$ with a SLAC mode launcher [9].

The large phase advance of $5 \pi / 6$ offers two main advantages: First, it means an intrinsically lower group velocity even at large apertures, providing a relatively high gradient and moderate required input power, and second, smaller transverse wakes, since a longer cell means a smaller number of irises per structure. The relevant parameters of the adopted $X$-band cavity are summarized in Table I.

The structure will compensate the second order nonlinearities in the single bunch phase space introduced by the $S$ band linac. With the stronger curvature of the $12 \mathrm{GHz}$ operating frequency, the beam needs to be decelerated by $30 \mathrm{MeV}$. The design uses an average gradient of $40 \mathrm{MV} / \mathrm{m}$ with a relatively constant distribution over the structure. The required input power is a modest $29 \mathrm{MW}$.
TABLE I. Specifications.

\begin{tabular}{lc}
\hline \hline Parameter & Value \\
\hline Beam voltage & $30 \mathrm{MeV}$ \\
Maximum power & $29 \mathrm{MW}$ \\
Iris diameter & $9.1 \mathrm{~mm}$ (average) \\
Wakefield monitors & up/downstream \\
Operating temperature & $40^{\circ} \mathrm{C}$ \\
Fill time & $100 \mathrm{~ns}$ \\
Repetition rate & $100 \mathrm{~Hz}$ \\
\hline \hline
\end{tabular}

Because of the constant gradient design choice, the iris diameter changes along the structure, the lower value being limited by the detrimental effects of the transverse shortrange wakefields. As can be seen in Fig. 1, the iris diameter changes linearly with the number of cells: The mean aperture at $9.1 \mathrm{~mm}$ makes it a relatively open structure.

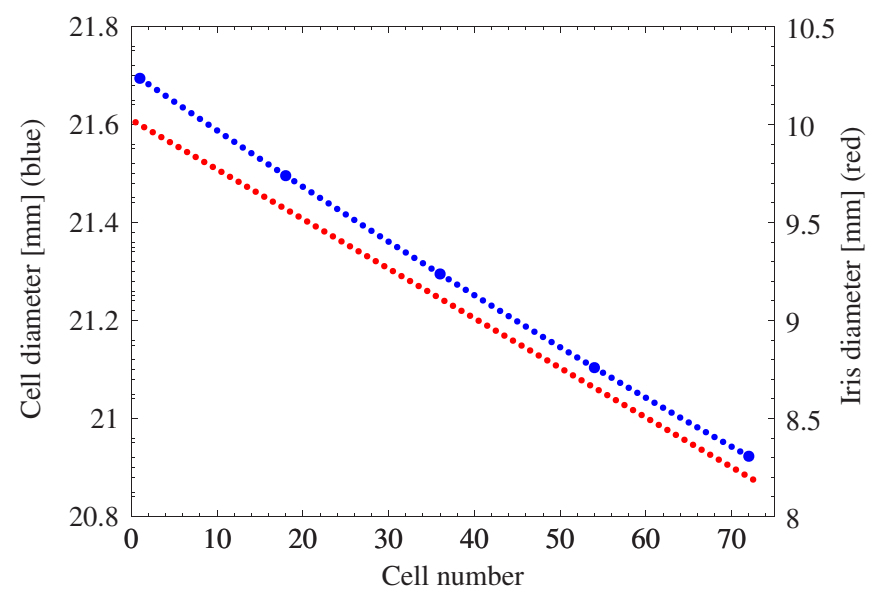

FIG. 1. (Color) Variation of the cell diameter and iris aperture along the structure omitting the monitor cells described later. The first cell has $9.986 \mathrm{~mm}$ iris diameter, the last one $8.239 \mathrm{~mm}$.

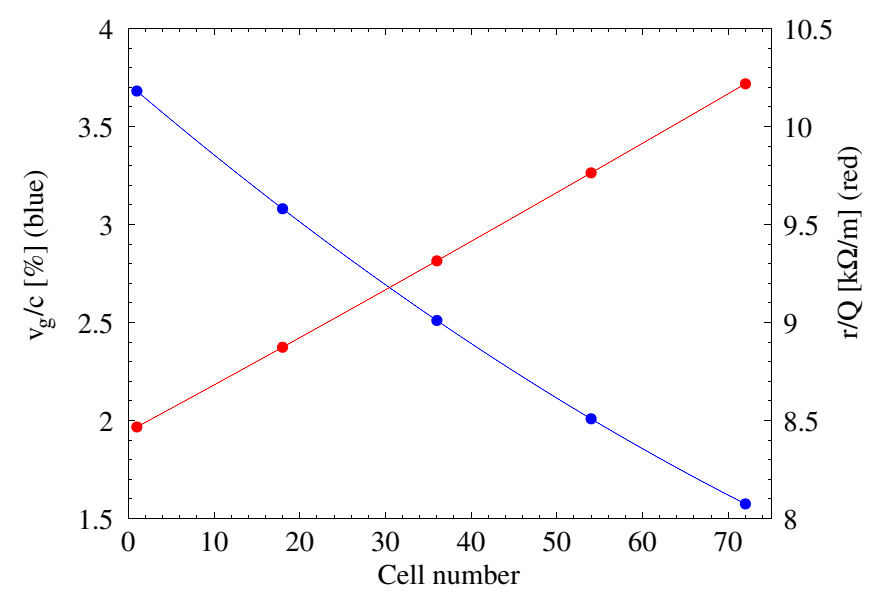

FIG. 2. (Color) Variation of group velocity and $r / Q$ along the structure. 


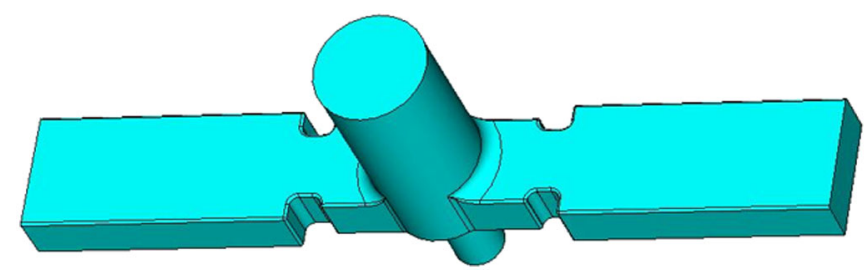

FIG. 3. (Color) The mode launcher geometry converting the waveguide mode into the circular $\mathrm{TM}_{01}$ mode at a match better than $-45 \mathrm{~dB}$.

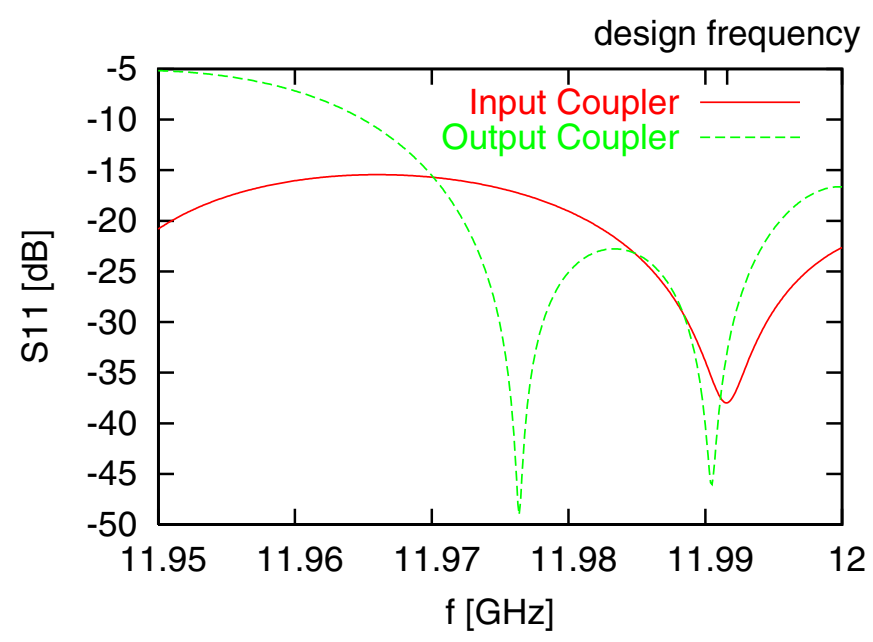

FIG. 4. (Color) Reflection coefficient for the input and output matching cells.

Concerning the group velocity variation, the $v_{g} / c$ is $3.7 \%$ at the level of the first regular cell and decreases to $1.6 \%$ at the last regular cell. The $r / Q$ value increases quasilinearly from $8.5 \mathrm{k} \Omega / \mathrm{m}$ to $10.2 \mathrm{k} \Omega / \mathrm{m}$ and the average $Q$ value computed is about 7150 . The variation of group velocity and of the $r / Q$ along the structure is shown in Fig. 2. The filling time of the structure is about $100 \mathrm{~ns}$.

The input and output coupler design is based on the SLAC mode launcher design [9], which converts a rectangular $\mathrm{TE}_{10}$ waveguide mode into the circular $\mathrm{TM}_{01}$ waveguide mode of the $X$-band structure (Fig. 3). The big advantage of this design is the reduced surface field, due to the rounded and thickened iris horns and the coupling of the power through the broad wall of the feed waveguide. The dimensions of the cells between the mode launcher and the structure ends are used to match the $\mathrm{TM}_{01}$ mode from the beam pipe into the accelerating structure. Figure 4 shows the reflection coefficients for the input and output part.

\section{WAKEFIELD MONITOR}

Beam offsets in an accelerator structure will generate transverse higher order modes with an amplitude proportional to the offset. It is attractive to use this effect to measure beam offset and structure alignment and various setups are currently in use.

One is a diagnostics system using the $1.3 \mathrm{GHz}$ superconducting cavities at FLASH $[10,11]$, where higher order mode signals from the nine cells in one of the cavities are obtained via two HOM couplers. Both longitudinal and transverse higher order modes are coupled out; the transverse signal is used to measure the alignment of the cavity and the longitudinal signal to give the beam phase.

Another setup uses wake signals from a damped, detuned NLC prototype [12]. Here the signal comes from the damping manifold, which contains only contributions from the dipole spectrum. A high quality spectral analysis of the lowest dipole band not only provides the global beam offset but also detailed information about the internal alignment of the structure.

The constant gradient design used here also has a distributed dipole spectrum, which may allow some of the same information to be seen, even if the bandwidth is somewhat reduced in comparison to a damped detuned structure. The important difference is the missing damping manifold collecting signals throughout the structure, so that we have to introduce a few coupler cells to extract the HOM signal.

Where to place the couplers, how to design them, and what to expect from the measurement are explained in the following.

\section{A. Location}

To understand the basic behavior of the long range wakes in such a structure, we look at the dispersion of the lowest dipole bands of a typical cell shown in Fig. 5. As in a classical $2 \pi / 3$ structure, we have hybrid HEM-type dipole modes; the lower dipole band starts at zero phase with a strong TE-type characteristic morphing into a TM characteristic as the phase approaches 180 degrees. At $5 \pi / 6$ the cell is longer, so the speed of light line and the

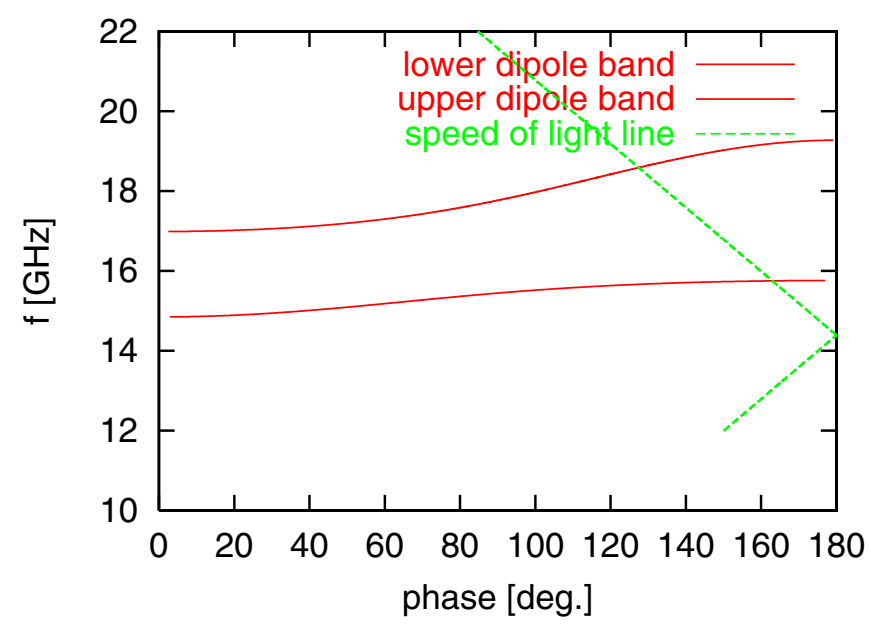

FIG. 5. (Color) Dispersion diagram of lowest two dipole bands in cell 36. 


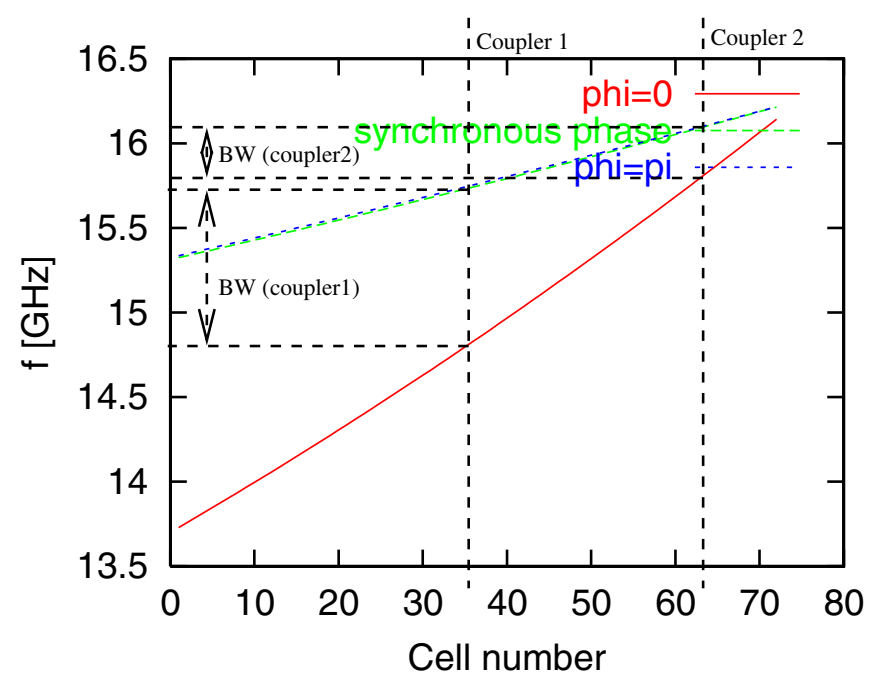

FIG. 6. (Color) Limits of lowest dipole band and synchronous frequency versus cell number. Also shown is the location of the wakefield couplers with their respective bandwidths.

dispersion curve are crossing at opposite slopes (this holds true for all cells in the design); the beam will excite a backward wave in the lowest dipole band. The synchronous phase is near 180 degrees.

The distribution of bandwidths and synchronous frequencies of the lowest dipole band inside the whole structure is shown in Fig. 6. We can identify three different frequency regions. Dipole modes with resonances in the region of 15.3 to $16.1 \mathrm{GHz}$ will be trapped modes, which are localized in only part of the structure, since cells at the beginning and the end cannot propagate these frequencies. These trapped modes start in one of the upstream cells with a field distribution corresponding to a $\pi$ mode and continue with a decreasing phase advance to the downstream cell, where it goes to zero. The excitation by the beam is most pronounced in the cell, where the resonance corresponds to the synchronous frequency and phase, which is close to 180 degrees. Modes with frequencies below $15.3 \mathrm{GHz}$ (the $\pi$ mode of cell 1) will extend from the input coupler to the cell where the phase advance goes to zero. Most of these modes will have only a weak kick factor, since their frequency does not correspond to one of the synchronous frequencies. The last region contains modes above $16.1 \mathrm{GHz}$; the modes extend into the output coupler and have a high kick.

With this in mind, we are able to choose suitable locations for the wakefield monitors. We assume that the wakefield monitor will couple only weakly to the resonant fields and will not distort the modal pattern inside the structure. Dipole wakes excited by offsets in the upstream half of the structure will correspond to modes which are concentrated in the middle of it. So we put the upstream monitor in cell 36. It will have a bandwidth of 14.8 to $15.7 \mathrm{GHz}$ (the width of the dipole band in cell 36). The corresponding modes have their synchronous phases in the upstream half and

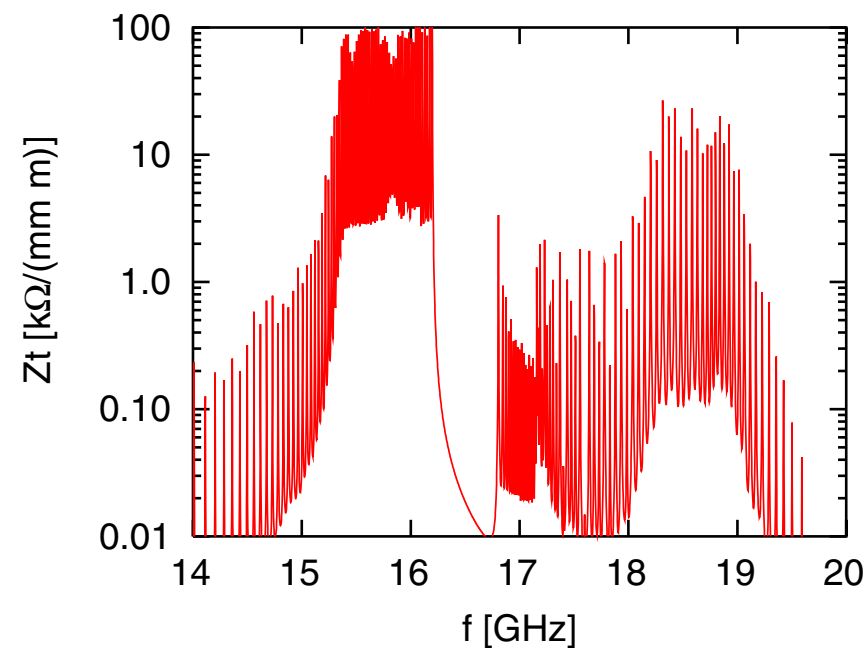

FIG. 7. (Color) Transverse wake impedance for the structure as calculated via the dual resonator model [13].

will signal offsets there. For the downstream coupler, we are restricted in the bandwidth of the cells - a reasonable compromise is to put it in cell 63 , which receives wakes excited in cells 40-63.

Wake monitor signals and wakes, to be shown in the following, were obtained from a dual resonator equivalent circuit, which is described in the Appendix. The transverse beam impedance computed for the whole 72 cell structure is shown in Fig. 7. As can be expected from the distribution of frequencies shown in Fig. 6, modes below $15.3 \mathrm{GHz}$ contribute only weakly to the overall wake. Only in the region from 15.3 to $16.2 \mathrm{GHz}$ do we see a strong coupling to the beam. The model takes account also of the Ohmic losses occurring in the structure, so the resonances show up with finite amplitude values in the beam impedance.

\section{B. Monitor design}

What are the criteria for the pickup to measure the transverse wakes? There should be a minimum perturbation of the fundamental mode. In order to effectively measure the position, the wake monitor should reject background signals coming from the fundamental as well as longitudinal higher order modes. These modes do not have azimuthal electric components and can be rejected by selectively coupling to the transverse electric components of the hybrid dipole modes. This can be obtained by a suitable geometrical orientation of the coupling waveguide. Any fundamental mode power still coupling due to geometrical misalignments is attenuated by the waveguide cutoff of approximately $13 \mathrm{GHz}$.

There are two different options for the coupling: One is to use radially oriented waveguides sitting head-on on the cavity wall and the other is to have a waveguide in parallel to the beam axis being side coupled - similar to the design of the damped detuned structure, but coupling only to one select cell. The side coupled version has the advantage that 


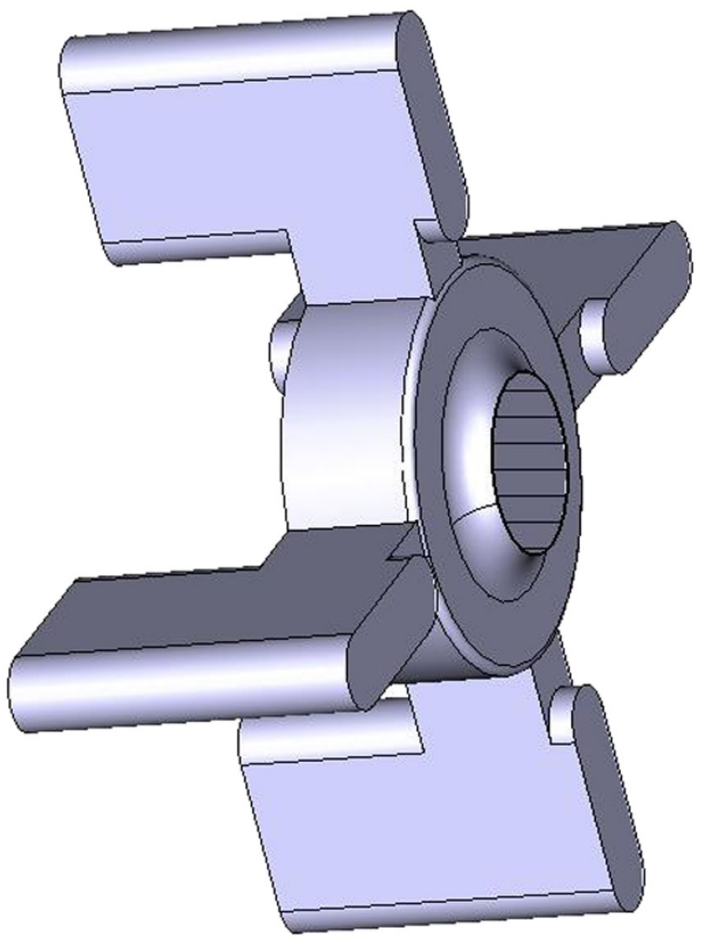

FIG. 8. (Color) Wake monitor cell using side coupled output waveguides with electric short on one side.

we can have a longer waveguide, damping down any residual signal from the fundamental mode. Also, that way there is enough space to integrate a transition from the waveguide to coaxial and to use a standard $50 \Omega$ vacuum feedthrough to connect the measurement front end. We chose this option. The design is similar to the HOM coupled cells of the damped detuned structures, the difference being that we couple only to one cell and have the waveguide electrically shorted on one side as shown in Fig. 8.

The coupling strength corresponds to a loaded $Q$ on the order of 800 at a phase advance of zero (where the lowest dipole band couples best) and gives a sufficient signal strength. As Table II shows, the effect on the fundamental mode is minor: introducing the coupler leads to $10 \%$

TABLE II. Parameters of monitor cells with and without wake coupler.

\begin{tabular}{lcccc}
\hline \hline & \multicolumn{2}{c}{ Cell 36 } & \multicolumn{2}{c}{ Cell 63 } \\
Monitor & Yes & No & Yes & No \\
\hline Fundamental & & & & \\
$r / Q(\mathrm{k} \Omega / \mathrm{m})$ & 9.29 & 9.3 & 9.97 & 9.97 \\
$Q$ & 6580 & 7180 & 6430 & 7025 \\
$v_{g} / c(\%)$ & 2.50 & 2.50 & 1.78 & 1.78 \\
Dipole mode & & & & \\
Start band $(\mathrm{GHz})$ & 14.80 & 14.83 & 15.76 & 15.80 \\
Stop band $(\mathrm{GHz})$ & 15.74 & 15.75 & 16.08 & 16.10 \\
Loaded $Q$ & 930 & N/A & 740 & N/A \\
\hline \hline
\end{tabular}

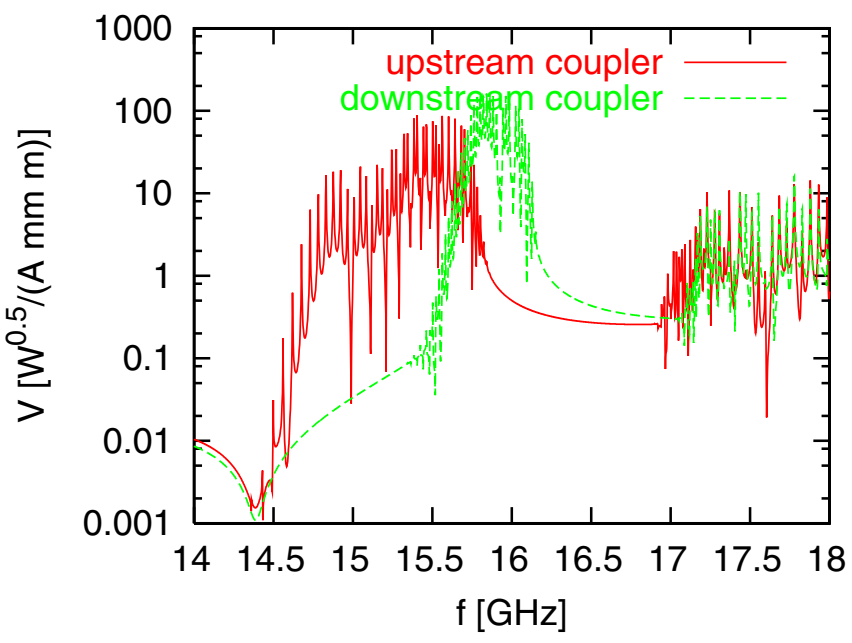

FIG. 9. (Color) Simulated output signals from upstream and downstream monitors versus frequency.

decrease in the $Q$ factor-the group velocity and the $r / Q$ stay virtually unchanged. The influences on the band limits of the lowest dipole band and also on the modal distribution of the structure are negligible.

\section{Signals}

Figure 9 shows the signal spectra computed for the upstream and downstream couplers. As can be expected from the previous discussion, we see two distinct frequency bands, corresponding to the synchronous frequencies in the two parts of the structure.

Before we look at other properties, one should explain in a little bit more detail, what is going on inside the structure. A dipole mode is excited by the beam dominantly in the "synchronous" cells, whose resonant frequency corresponds to the synchronous one. The mode does not build up instantaneously, but the energy deposited by the beam in this synchronous cell needs time to get distributed in the

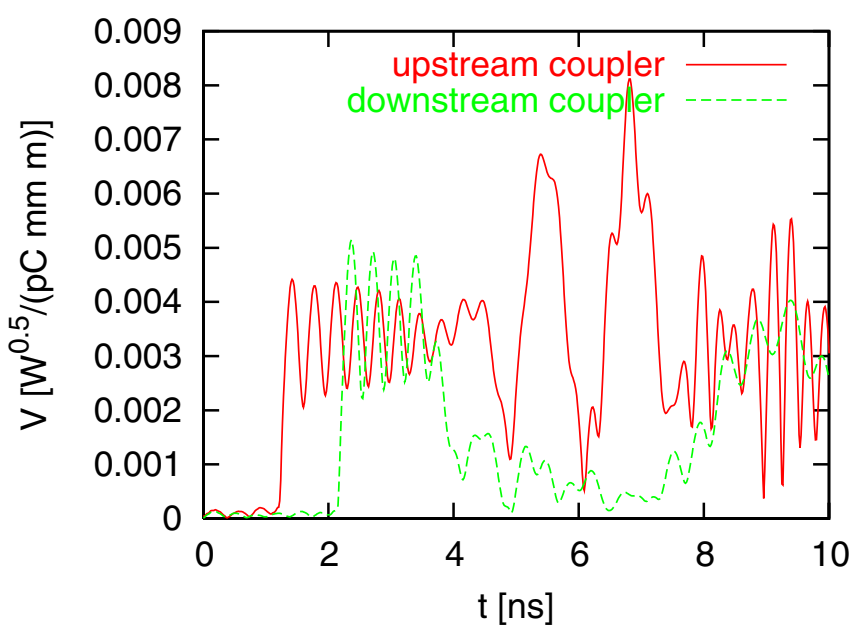

FIG. 10. (Color) Signal envelope from upstream and downstream monitors vs time. 


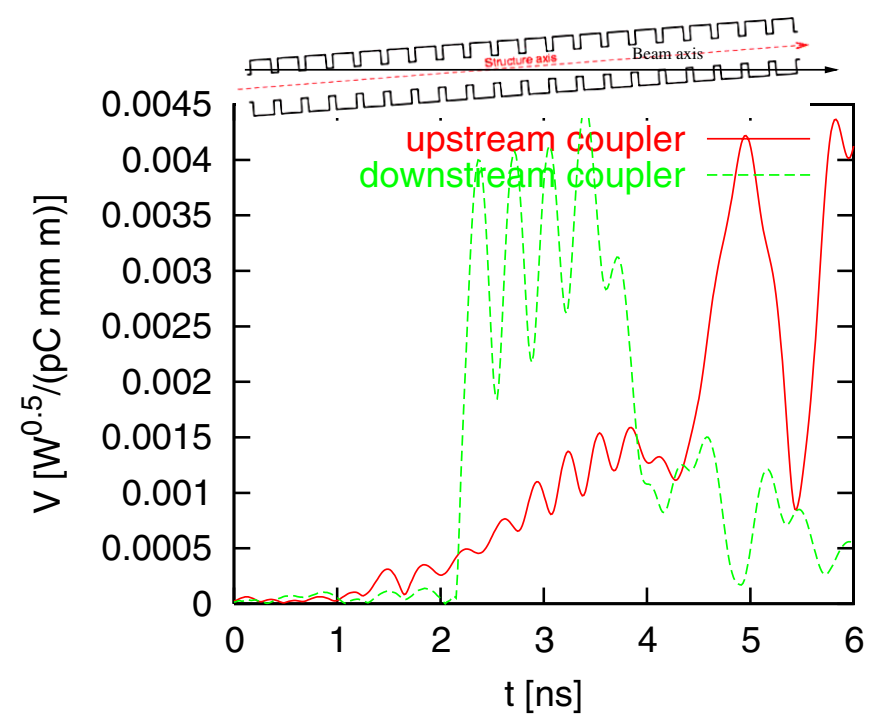

FIG. 11. (Color) Signal envelope upstream and downstream monitors vs time assuming structure axis tilted to beam axis.

structure- the distance between the synchronous cell and the wakefield monitor leads to a time delay, before the signal shows up in the monitor signal. Signals from different parts of the structure have different delays. In the time domain (Fig. 10), this shows up as an initial rectangular pulse (with some beating on top) of 2-3 ns length.

We can push the analysis still further. The beam offset in individual parts of the structure is correlated to different synchronous frequencies, and this in turn with different delays in the time domain signal. So one could question whether it is possible to also identify tilts with respect to the beam. In this case, the middle of the structure is aligned with the beam axis, whereas the extremities would be offset generating a transverse signal. The resultant signals are shown in Fig. 11. The upstream monitor is the more significant one in this case, since it sees the whole front half of the structure. Where a constant offset gives a more or less rectangular pulse as in Fig. 10, we see now a ramp, starting at zero (no offset in the middle) and growing linearly to the peak (high offset at beginning). The downstream signal shows a similar slope, which is not as pronounced, since it sees only part of the structure.

If one looks at still finer details such as a bend in the structure, using only the envelope of the signal does not work anymore, as can be seen in Fig. 12. The reason is the dispersion, which causes frequency dependent signal velocities and smears out the signal. To detect these, a more complex spectral analysis of the signals would be required.

An important question is the resolution of the monitors. Apart from the usual considerations-thermal noise in the pickup and front end and spurious signals from other sources-we have a significant contribution from the random uncorrelated misalignment of individual cells. As the beam passes through the structure seeing these random offsets, it will, also with a perfect global alignment, excite

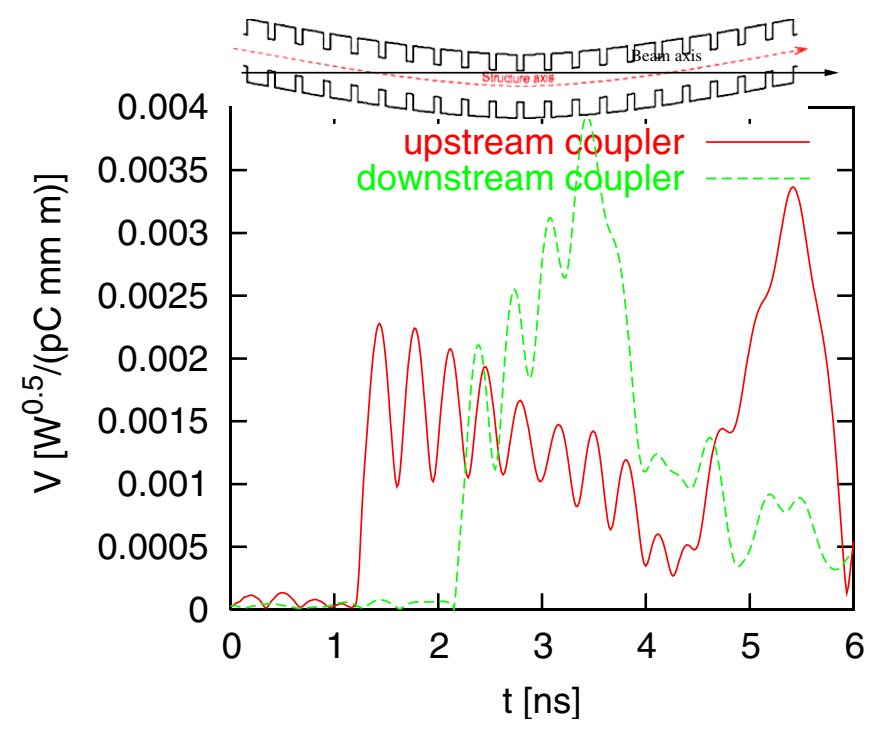

FIG. 12. (Color) Signal envelope upstream and downstream monitors vs time assuming bent structure.

wakefields and wake signals, which we will see as a noisy background to the measurement. To estimate this contribution, we included this random offset model into the equivalent circuit and simulated it. Figure 13 shows an example for the output signal. Comparing the peak random offset signal in the first four nanoseconds, roughly $0.008 \mathrm{~W}^{0.5} / \mathrm{pC} \mathrm{mmm}$, with the constant offset signal (Fig. 10) of 0.004 gives the impact on the measurement. A structure with a random cell-to-cell offset of $1 \mu \mathrm{m} \mathrm{rms}$ will create a signal proportional to a systematic offset of $2 \mu \mathrm{m}$; the wake monitors will have a resolution limit of $2 \mu \mathrm{m}$. We would like to see resolutions below $10 \mu \mathrm{m}$,

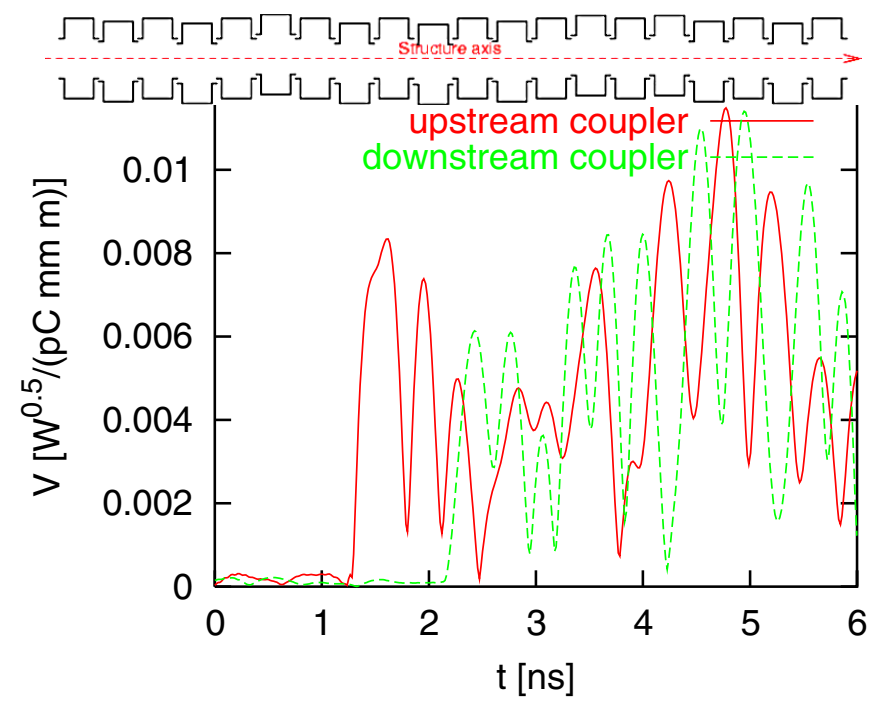

FIG. 13. (Color) Signal envelope upstream and downstream monitors vs time assuming random, uncorrelated misalignment of individual cells in the structure. The signal value is normalized to the standard deviation of the misalignments ( $1 \mathrm{~mm}$ here). 
which corresponds to a mechanical cell-to-cell alignment of better than $5 \mu \mathrm{m}$, which is entirely feasible.

In principle, one would expect a similar time domain behavior also from the signal of a damped detuned structure as in [13], but here the propagation velocity of the electromagnetic fields in the manifold is relatively high, which favors a spectral analysis of the signal. Our design, on the other hands, profits from the low group velocity of the dipole bands within the structure, stretching the time domain response and allowing one to profit from the correlation between signal frequency and signal delay to measure offsets and misalignments straightforward using the signal envelope.

\section{CONCLUSION AND OUTLOOK}

A newly designed $X$-band structure for the new CLIC reference frequency has been presented. It is to be used for high gradient testing at CLIC and as a prototype structure for the PSI-XFEL injector. It offers an efficient fundamental mode performance with a wide aperture minimizing transverse wakefield effects. It includes two wakefield monitor cells measuring offsets in the upstream and downstream parts of the structure, respectively. Misalignment of parts of the structure show up as a distinct pattern in the envelope of the time domain signal, so that the use of a relatively primitive detector $\mathrm{rf}$ front end should be possible for operation in single bunch mode. The expected resolution is mainly determined by tolerances in the mechanical cell-to-cell alignment. Values below $10 \mu \mathrm{m}$ seem to be entirely feasible.

With the rf design finished, we are currently in the transition to the mechanical design, which will be followed by manufacturing and low level rf tests. Structures for high power testing are expected to be ready in autumn 2009.

\section{APPENDIX A: SIMULATION OF DIPOLE HIGHER ORDER MODES}

With modern high performance codes, the collective behavior of multicell structures can be simulated. Nonetheless, given their fast speed and their comprehensiveness, approximate models using equivalent circuits can be very useful for design purposes. This is especially true for the dual band model described below, which has been validated extensively [14].

The main contribution to the transverse wake comes from lowest dipole band and so considerable effort was put into an analysis of the response, which was not possible with the computer codes at that time. A very first approach assumed uncoupled cells each contributing a kick at its synchronous frequency using values computed from numerical simulation of the individual cells with, for example, TRANSVRS or MAFIA. The method gives a good approximation of the global frequency distribution of the beam impedance determining the roll-off of the wake, but falls short in describing the modal density responsible for

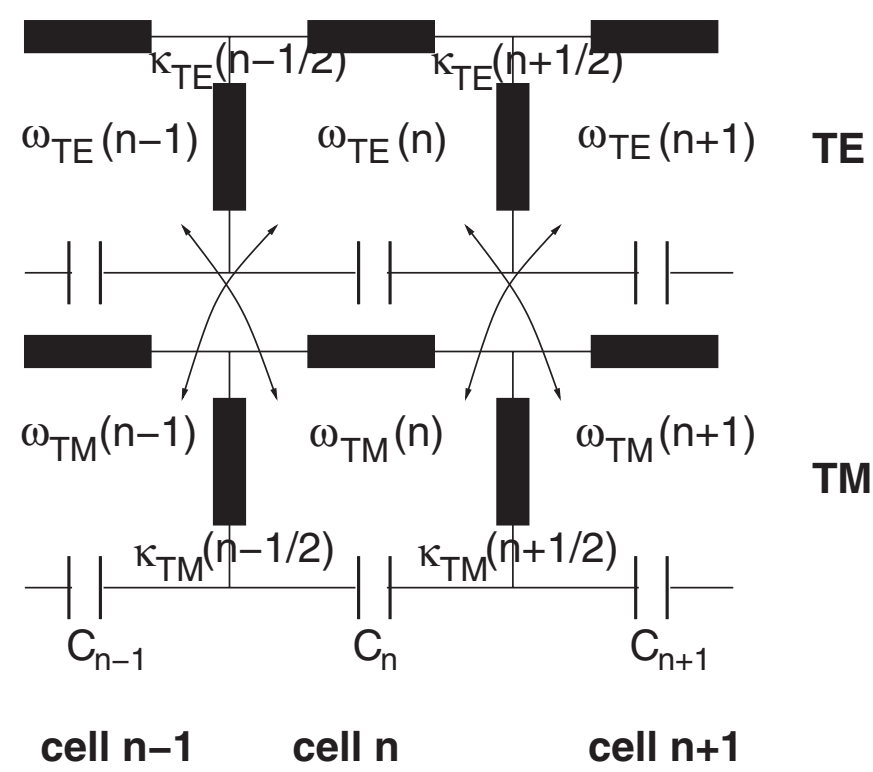

FIG. 14. Double resonator chain used to model the collective wake in the lowest two dipole bands. It is fully characterized by the resonant frequencies and cell-to-cell couplings $\omega$ and $\kappa$ for the TE and TM parts as well as the TM capacitor giving the coupling to the beam.

recoherence effects, so that results for the long range wake are inaccurate.

The next step was the introduction of a coupled resonator chain [15], which improved the quality of the long range wakes quite a bit, but was nonphysical in the description of the kick factor. These problems were solved by expanding the circuit into a double resonator chain as in Fig. 14, where the chains model the transverse, respectively magnetic parts of the hybrid transverse mode [16]. Not only does the variation of the kick factor versus phase advance become more precise, but also two dipole bands instead of only one are modeled.

With inverse resonances $x_{m}=1 / \omega_{\mathrm{TE}}^{2}, \tilde{x}_{m}=1 / \omega_{\mathrm{TM}}^{2}$, cell couplings $\kappa_{m+(1 / 2)}, \tilde{\kappa}_{m+(1 / 2)}$, and cross couplings $\breve{\kappa}_{m+(1 / 2)}=\sqrt{\kappa_{m+(1 / 2)} \tilde{\kappa}_{m+(1 / 2)}}$, one gets the following circuit equations:

$$
\begin{gathered}
\left(x_{m}-\lambda\right) a_{m}-\frac{\boldsymbol{\kappa}_{m+(1 / 2)}}{2} a_{m+1}-\frac{\boldsymbol{\kappa}_{m-(1 / 2)}}{2} a_{m-1} \\
+\frac{\breve{\kappa}_{m+(1 / 2)}}{2} \tilde{a}_{m+1}-\frac{\breve{\kappa}_{m-(1 / 2)}}{2} \tilde{a}_{m-1}=0 \\
\left(\tilde{x}_{m}-\lambda\right) \tilde{a}_{m}-\frac{\tilde{\kappa}_{m+(1 / 2)}}{2} \tilde{a}_{m+1}-\frac{\tilde{\kappa}_{m-(1 / 2)}}{2} \tilde{a}_{m-1} \\
-\frac{\breve{\kappa}_{m+(1 / 2)}}{2} a_{m+1}+\frac{\breve{\kappa}_{m-(1 / 2)}}{2} a_{m-1}=b_{m} .
\end{gathered}
$$

$\lambda=\frac{1}{\omega^{2}}$ is the frequency, $b_{m}$ the driving term given by beam current times cell offset, and $a_{m}$ and $\tilde{a}_{m}$ the amplitudes in 
the TE and TM circuits, respectively. As in the other methods, dispersion and kick factors are computed numerically for the geometry of individual cells, for which the resonance and coupling terms in the equations are fitted. (For more information see $[13,16]$.) An important extension for NLC/GLC type damped detuned structures included an additional parametrization of the damping manifold [13]. For structures with dedicated HOM loads [17], there is also an adapted model [18] allowing the inclusion of nonideal waveguide loads in the circuit.

For the structure presented here, we essentially stayed with the simple double-band model with the following minor changes. Given that we are mainly interested in the signal of the wakefield monitors coupling only to trapped modes inside the structure, we modeled only the cells omitting the power couplers and their effect on the transverse mode spectrum. Internal losses in the structure were modeled by introducing a complex resonance frequency:

$$
\begin{gathered}
\hat{\omega}_{\mathrm{TE}}=\omega_{\mathrm{TE}}\left(1-\frac{j}{2 Q_{\mathrm{TE}}}\right) \\
\hat{\omega}_{\mathrm{TM}}=\omega_{\mathrm{TM}}\left(1-\frac{j}{2 Q_{\mathrm{TM}}}\right) .
\end{gathered}
$$

We expect a good match for output of the wakefield monitors and, in addition, they do not affect the cell-tocell coupling like the DDS manifold, so it is sufficient to describe their effect on the modal spectrum by the change in resonant frequencies and coupling and by the loaded $Q$ factor $Q_{l}$ seen by the TE resonant circuit; beam impedance and wakes are obtained by directly solving the inhomogeneous equations using the spectral function method de-

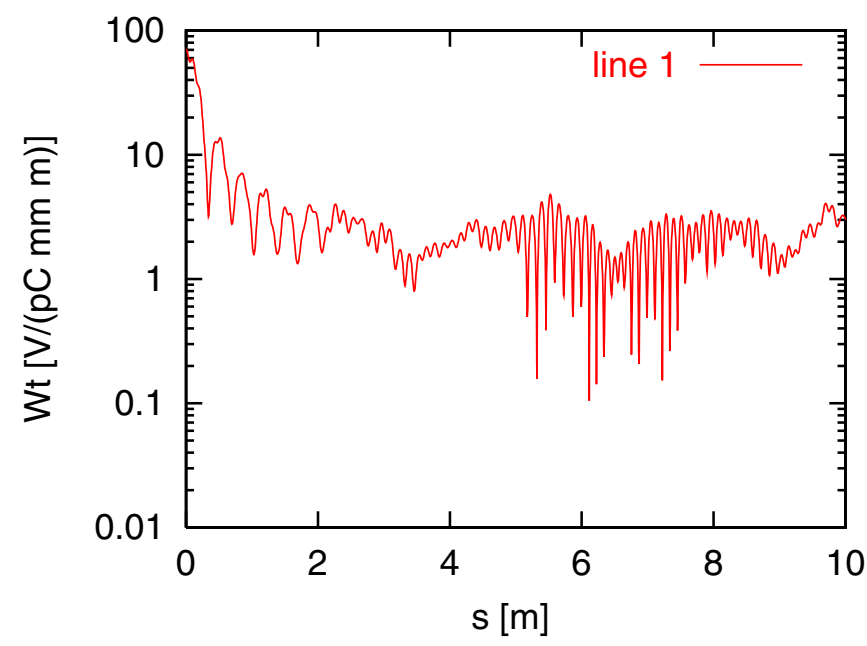

FIG. 15. (Color) Transverse wake function of $X$-band structure. scribed in [19]. The transverse impedance is shown in Fig. 5 and the corresponding wake in Fig. 15.

The output signal from the monitors is obtained directly from the computed amplitudes $a(\omega)$ of the equivalent TE resonator in the monitor cell. The energy $W_{\mathrm{TE}}$ stored in the TE resonator is proportional to $a^{2}(\omega)$ and the loaded $Q$ gives the extracted power as

$$
P_{\text {out }}(\omega)=\left|v_{\text {out }}(\omega)\right|^{2}=\frac{\omega W_{\mathrm{TE}}}{Q_{l}} \sim \frac{\omega a^{2}(\omega)}{Q_{l}} .
$$

For the calculation of the time domain responses, the complex phases of the output signals $v_{\text {out }}(\omega)$ are assumed to follow closely those of the resonator amplitudes $a(\omega)$. Apart from a constant phase jump, we have frequency dependent time delays introduced by coupling irises and waveguides, but these are minor compared to the nanosecond scale duration of the output pulses.

Nonconstant offsets as encountered in tilted or bent structures can be introduced conveniently in the driving term $b_{m}$ of the circuit equations, which apart from the kick factor also contain the beam offset in cell $\mathrm{m}$. Tilt and bend effects were normalized to the maximum offsets, whereas for the random cell-to-cell misalignment a Gaussian distribution was assumed.

[1] C. E. Adolphsen et al., SLAC Reports No. SLAC-R-559, SLAC-R-0559, SLAC-559, SLAC-0559, KEK Report No. KEK-REPORT-2000-7, LCC Report No. LCC-0042, 2000, p. 286, ISG Progress Report, 2000.

[2] J. N. Galayda et al., in the 21st International Linac Conference (LINAC 2002), Gyeongju, Korea, 2002, p. 6 (SLAC-PUB-10115).

[3] C. Vaccarezza et al., in Proceedings of the 2007 Particle Accelerator Conference, Albuquerque, New Mexico, 2007 (IEEE, Albuquerque, New Mexico, 2007) (SLAC Report No. SLAC-PUB-12963, 2007).

[4] C. J. Bocchetta et al., Report No. ST/F-TN-07/12, 2007, http://www.elettra.trieste.it/docs/FERMI_elettra-CDR. pdf.

[5] A. Oppelt et al., in Proceedings of the 2007 Free-Electron Laser Conference (FEL2007), Novosibirsk, Russia, p. 224.

[6] R. Zennaro, "Linacs for Hadrontherapy: CABOTO, a $X$-band CArbon BOoster for Therapy in Oncology," Proceedings of the $X$-Band RF Structure and Beam Dynamics Workshop-44th ICFA Advanced Beam Dynamics Workshop, 2008, Daresbury, UK (to be published).

[7] D. Sprehn et al., in the SPIE 2000 AeroSencse Symposium, Orlando, Florida, 2000.

[8] http://lcdev.kek.jp/ISG/ISG9.ZL2.pdf.

[9] C. Nantista, S. Tantawi, and V. Dolgashev, Phys. Rev. ST Accel. Beams 7, 072001 (2004).

[10] J. Frisch et al., in Proceedings of the 10th European Particle Accelerator Conference, Edinburgh, Scotland, 2006 (EPS-AG, Edinburgh, Scotland, 2006), pp. 920-924.

[11] S. Molloy et al., Meas. Sci. Technol. 18, 2314 (2007). 
[12] M. Seidel, in Proceedings of the Particle Accelerator Conference, Vancouver, BC, Canada, 1997 (IEEE, New York, 1997), ISBN 0-7803-4376-X, pp. 434-438.

[13] R. M. Jones, C. E. Adolphsen, J. W. Wang, and Z. Li, Phys. Rev. ST Accel. Beams 9, 102001 (2006).

[14] R. M. Jones et al., New J. Phys. 11, 033013 (2009).

[15] M. Drevlak, Ph.D. thesis, Technische Hochschule Darmstadt, Darmstadt, Germany, 1991.

[16] K. L.F. Bane and R.L. Gluckstern, SLAC Report No. SLAC-PUB-5783, 1992.

[17] I. Wilson et al., SLAC Report No. SLAC-PUB-8941,
CERN Report No. CERN-PS-2000-044, CLIC Report No. CLIC-NOTE-443, 2001, p. 4; also "Vienna 2000, EPAC 00," in Proceedings of the 7th European Particle Accelerator Conference (EPAC 2000), Vienna, Austria, 2000, pp. 516-518.

[18] M. Dehler, CERN Report No. 98-09.

[19] R. M. Jones, N. M. Kroll, and R. H. Miller, in Proceedings of the 18th International Linear Accelerator Conference (Linac 96), Geneva, Switzerland, 1996, pp. 650-652 (SLAC-PUB-7287). 